\title{
GAME THEORY BASED INTERFERENCE CONTROL AND POWER CONTROL FOR D2D COMMUNICATION IN CELLULAR NETWORKS
}

\author{
Fa-Bin $\mathrm{Li}^{1}$, Peng-Xiang $\mathrm{Li}^{2}$ Ya-Fei Yang ${ }^{2}$ and Hui $\mathrm{Li}^{2 *}$ \\ ${ }^{1} 63812$ Troop of PLA, Wenchang, P. R. China \\ ${ }^{2}$ College of Information Science and Technology, Hainan University, Haikou P. R. China
}

\begin{abstract}
With the current development of mobile communication services, people need personal communication of high speed, excellent service, high quality and low latency, however, limited spectrum resources become the most important factor to hamper improvement of cellular systems. As big amount of data traffic will cause greater local consumption of spectrum resources, future networks are required to have appropriate techniques to better support such forms of communication. D2D (Device-to-device) communication technology in a cellular network makes full use of spectrum resources underlaying, reduces the load of the base station, minimizes transmit power of the terminals and the base stations, thereby enhances the overall throughput of the networks. Due to the use of multiplexing D2D UE (User equipment) resources and spectrum, and the interference caused by the sharing of resources between adjacent cells, it has become a major factor affecting coexisting of cellular subscribers and D2D users. When D2D communication multiplexes the uplink resources, the base-stations are easily to be disturbed; when the downlink resources are multiplexed, the users of downlink are susceptible to interference. In order to build a high-efficient mobile network, we can meet the QoS requirements by controlling the power to suppress the interference between the base station and a terminal user.
\end{abstract}

\section{KEYWORDS}

Cellular Network, Device-to-device, Mode Selection, Power Control, Interference Control

\section{INTRODUCTION}

As one of the key techniques of the next generation wireless communication systems, the long term evolution (LTE) of the Third Generation Partnership Project (3GPP) is committed to provide technology for high data rates and system capacity. In addition, advanced LTE (LTE-A) was defined to support new components for LTE to meet the higher communication requirements. Local area services are considered as a hot topic in evolution, and by reusing spectrum resources local data transfer rate have been increased dramatically. However, the unlicensed spectrum reuse may bring inconvenience for local service provider to guarantee a stable control environment, such as, WiFi and Bluetooth. It does not provide the security and quality of service (QoS) like cellular networks. The maximum reason is that the use of unlicensed band cell interference can be controlled, such as in the Ad hoc network, it is not in the control of base station or other central node. Therefore, the method of accessing the licensed spectrum has attracted much attention. 
Device-to-device (D2D) communication is a promising technical component for LTE-A. The existing studies allow D2D as the underlying cellular network technologies to increase the spectral efficiency. In a D2D communication, the user equipment (UE) transmits signals to each other over a direct link using the cellular resources instead of through the base station. This is different from the femtocell, whose users communicate with the help of small low-power cellular base stations. D2D user communicates directly but remains controlled under the base station. Therefore, the potential of improving spectral utilization has promoted much work in recent years, which shows that D2D can improve system performances by reusing cellular resources. As a result, D2D is expected to be a key feature supported by next-generation cellular networks.

Although D2D communication brings improvement in spectrum efficiency and makes large benefits on system capacity, it causes interference to the cellular network as a result of spectrum sharing. Thus, an effective interference coordination scheme must be configured to ensure that the targeted performance level of the cellular communication. There exists a lot of work to be done about the power control of D2D user equipment to limits co-channel interference ${ }^{[1]}$. Janis et al. use Multiple Input and Multiple Output (MIMO) transmission scheme in order to avoid interference from cellular downlink to D2D receives for sharing the same resources, which aims at guaranteeing the D2D performance ${ }^{[2]}$. Methods of interference management both from cellular network to D2D communication and from D2D to the cellular network have been proposed ${ }^{[3]}$. In order to further improve the gain from intra-cell the spectrum reuse, Janis et al. studied the appropriate matching problem between the cellular and D2D user when sharing the same resources $^{[4]}$. Zulhasnine et al. proposed an alternative greedy heuristic algorithm to lessen interference to the primary cellular networks with channel state information $(\mathrm{CSI})^{[5]}$. But this scheme is easy-operated but cannot prevent huge signalling overhead. In manuscript [6], the resource allocation scheme avoids the harmful interference by tracking the near-far interference, identifies the interfering cellular users and allows uplink frequency band effectively used, whose goal is to avoid interference from the cellular to the D2D communication. In the literature [7], Yu et al. first provided optimal resource allocation and power control between cellular and D2D communication that share the same resources under different resource sharing modes, and evaluated the performance of the D2D underlay system in both a single cell scenario and a Manhattan grid environment. Then, they further optimized the resources usage among users sharing the same resources. These studies mentioned above shows that through proper resource management, D2D communication can improve system throughput with restricted interference between cellular networks and D2D transmissions as shown in Figure 1. However, the problem of allocating cellular resources to D2D transmissions is of great complexity.
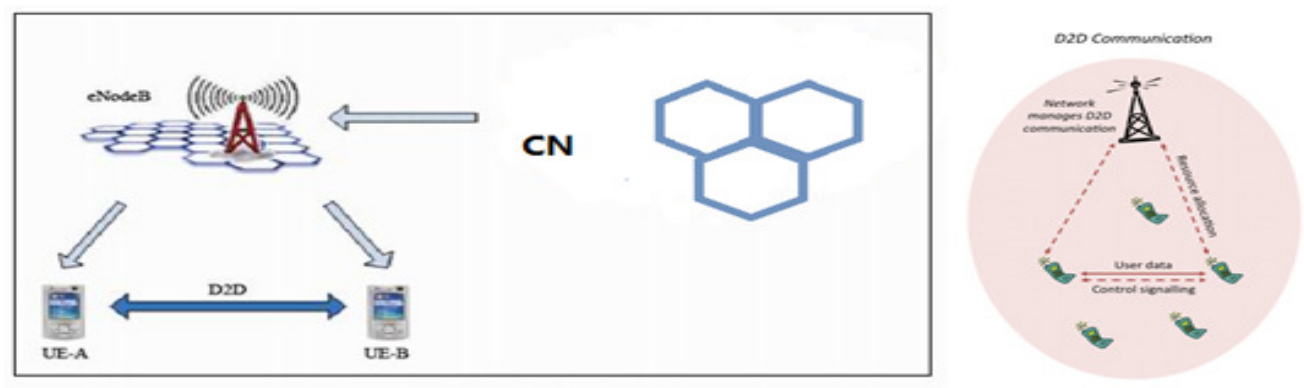

Figure 1. Network management in the cellular networks with D2D communication 


\section{SELECTION OF THE COMMUNICATION MODES}

In D2D underlying communication system, there are many problems related to the channel, one of which is to determine whether one should use a cellular communications mode or the direct communication mode. In D2D mode, data is transmitted directly to the receiver, but the cellular communication mode requires that the source device transmits data to the base station and the destination devices use base station downlink. Here we consider three different modes of selection criteria:

1. Cellular mode: All devices use a base station to communicate.

2. Dedicate Mode: D2D user pairs occupy specialized cellular band.

3. Reuse mode: D2D users and cellular users to share cell resources.
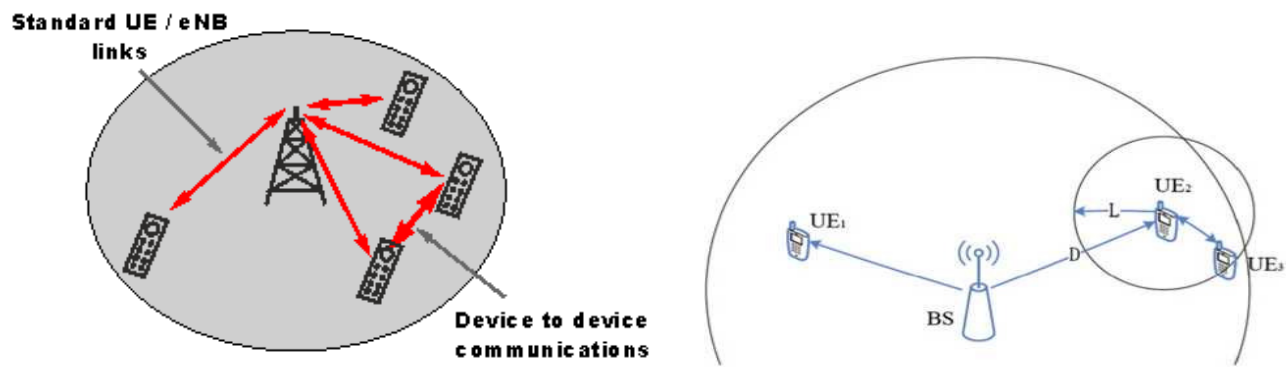

Figure 2. D2D communication in the cellular networks

D2D mode is selected if any of the path losses between source device and its serving base station, and between the target device and its serving base station, is greater than the path loss between a source and a destination in a pair. With the increase of the maximum distance between a pair of user, direct communication performance will decrease. Setting a threshold value to decide whether to use D2D communications, and multiplexing mode is a way to solve the inter-terminal communication overhead. From the perspective of cellular and D2D communication, choosing a better channel conditions may obtain an optimal performance. The results demonstrated that when the distance between cellular users and D2D user is near, the dedicated mode is best; when it is far or D2D user pairs are near, reuse mode is best as shown in Figure 2.

\section{GAME THEORY IN D2D COMMUNICATION}

Due to the interference caused by spectrum resources shared between D2D users and cellular users, resource management becomes a key problem to be solved. Game theory provides a set of mathematical tools to study the complex interactions among interdependence rational players and to predict their selection policy. In recent years, game theory has become a tool for the design of wireless communication networks. Therefore, we employ game theory in D2D communication for resource scheduling and interference avoidance.

The key elements of the game are the players, the actions, utilities and information, known as the rules of the game. The individuals making decision are called players, who can be represented as a set of $\mathbf{M}=\{1,2, \ldots, m\}$. An action $a_{i}$ is a choice that player $i$ makes. An action profile $\mathbf{A}=\left\{a_{i}\right.$ । $i \in M\}$ is a set of all the players actions. In the auction, the players are the bidder and actions are the bids submitted by bidders. Player $i$ 's earnings $U_{i}(\mathbf{A})$ is a function of the action profile $\mathbf{A}$, and 
utility describe how much gains the player gets from the game for every possible action profile. In the games, the players utility equals his value for the action profile $v_{i}(\mathbf{A})$ minus his payment $c_{i}(\mathbf{A})$ that $u_{i}(\mathbf{A})=v_{i}(\mathbf{A})-c_{i}(\mathbf{A})$. An important assumption of game theory is that all the players are rational, and they are going to choose to action to maximize their utilities. The players information can be characterized by an information set, which tells what kind of knowledge the players decide instance. In order to maximize their utilities, the players would design their strategies, which are mappings from player's information sets to his actions.

A reasonable prediction result of a game's outcome is equilibrium, in which each player to choose a best strategy to maximize his utility. Among several equilibrium concepts, we mainly put emphasis on the Nash equilibrium (NE). In a static game, Nash equilibrium is a strategy profile where no player can increase his utility by unilaterally change.

In the present study, game theory including a number of different methods is used for analysis resource allocation problem, for example, power and wireless spectrum allocations in communication networks ${ }^{[8]}$, resource management in $\operatorname{grid}^{[9]}$, and distributed resource coordination in large-scale container terminal ${ }^{[10]}$. In the literature [8], Bae et al. proposed a sequential auction method for sharing the wireless resources, spectrum management agent by the use of the program for a sequential second price auction collect bids and allocation discrete resource unit.

Resource management cooperation auction model was introduced in [10]. Auction cooperation resource allocation mechanism is to allow resource cooperation bidders to place the bid, called package, rather than just the individual resource units. In fact, the cooperation auction (CA) has been applied in a variety of industries, such as trucking, airline arrival and departure slots, and the use of wireless communications services. Auction theory benchmark environment is a private value model, introduce by Vickrey, in which one bidder has a value for each package of items and the value is not related to the private information of other bidders ${ }^{[11]}$. Bidders do not realize a lot of work, care for their complex ways competing projects. The cooperation incentive auction agents fully express their preference, which is an advantage in improving system efficiency and auction profitability. Our interests are the application of cooperation auction game to resolve the problem of using the same cellular band by any D2D links and the optimization of system capacity.

However, cooperation in the auction game that there is a series of problems and challenges, such as pricing and bidding rules. The winner determination problem (WDP) always leads to the allocation of NP-hard. Therefore, we focus on iteration cooperation auction (I-CA) of game evolutionary mechanisms ${ }^{[12,13]}$. In the I-CA, the agents claim more iterative bidding, and bidders calculate temporary assignment and ask each round of the auction price.

We studied the underlying D2D communication based on a valid I-CA spectrum resources allocation to further improve system efficiency. The system consists of a base-station, multiple cellular users receiving signal from the base-station and D2D pairs using licensed spectrum resources to communicate with respective receives. Since the interference minimization is a key point and multiple D2D pairs sharing the same resources may bring greater efficiency on the system capacity, we think it has become a reverse I-CA Game. This means that agents may obtain opportunity in the resource competition. But the D2D links wait to be sold as goods or services. By this way, the packages of D2D pairs are auction off in each auction round. In addition, we study some important properties of the proposed resource allocation mechanism such as cheatproof, convergence and price-monotonicity. We reduce the computational complexity and apply the scheme to WINNER II channel models which contain a well-known interior scenario. 
Compared to other programs on the system, the auction algorithm has a good performance in total rate. It can improve the system efficiency while having lower complexity than the exhaustive searching allocation.

Previously works considered not much about time-domain scheduling of D2D communication. We use game theory approach to study the joint scheduling, power control and channel assignment for D2D communication. Note that if the cellular and D2D user are simply modelled as selfish players, the result is usually inefficient, because the cellular users do not want share channels with D2D users. When D2D communication is the main underlying cellular network, the principles of Stackelberg game is very suitable for the system. Stackelberg game is a hierarchical tiered system, and it has leaders and followers. Leaders acts first, then followers observe leader's behaviour and decides his strategy. The stackelberg game has been used in collaborative networks ${ }^{[14]}$ and cognitive radio networks ${ }^{[15,16]}$. In our proposed Stackelberg game, the cellular UEs is regarded as a leader and D2D UEs are followers. Our group cellular and D2D UEs from leaderfollowers pairs, and the leaders charges some fees on the followers for using the channel. We analysed the optimum price for the leader and the optional power for the follower. These policies lead to Stackelberg equilibrium. Then, we propose a joint scheduling and resource allocation algorithm. Leader-followers pairs form a priority queue base on their utilities, and the system can schedule the D2D UEs according to their order in the queue.

Flourish wireless services needs more power from users' batteries. However, UEs are typical hand-held devices with limited battery lifetime. Users have to constantly charge their batteries. A major advantage of D2D communication is to reduce users transmit power consumption and thus extend battery life. We consider the energy consumption of UEs' the circuit and transportation, and model the battery lifetime by using the Peukerts's law ${ }^{[17]}$. Our problem is to maximize battery lifetime of D2D UEs subject to a rate constraint.

\section{POWER CONTROL OF D2D COMMUNICATION}

The rapid growth of data service in wireless communication makes the shortage of spectrum resources, which becomes a challenge for mobile communications. It promotes the emergence of some advanced wireless networks and systems, aiming to provide a high rate and high quality communication network for a large number of users. In the traditional cellular networks, direct communication between users is not allowed. The communication process consists of two stages: one is the transmitter to the base station, namely the uplink; another is the base station to the receiver, namely the downlink. This centralized operation mode is convenient for the management and control of resources and interference, but its efficiency of resource utilization is low. In order to improve the efficiency of spectrum utilization, in 2008, the Qualcomm Corp first proposed the communication technology ${ }^{[18 \sim 20]}$ of D2D. In recent years, D2D communication has aroused wide attention. NOKIA, Ericsson, HUAWEI and so on has been committed to the research of this technology. D2D communication is a new technology that allows the end users to communicate directly through the shared cell resources under the control of the cellular system, as shown in Figure 3. The traditional cellular network ${ }^{[21 \sim 22]}$ transmits signals from transmitter to the base station, and then from the base station to the receiver, as shown in Figure 4. 


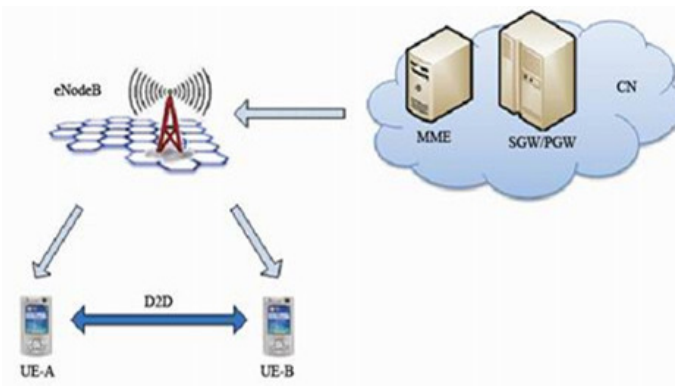

Figure 3. D2D communication network architecture

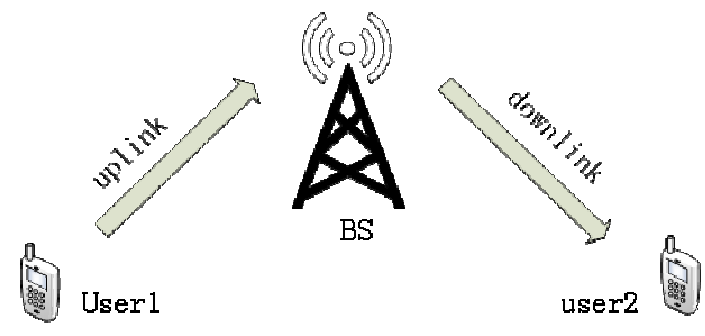

Figure 4. Traditional cellular network communication

This centralized control mode has low efficiency of resource utilization. Cellular network introduces D2D communication, which can reduce the burden of the base station ${ }^{[23,24]}$ and also reduce the communication delay ${ }^{[23,25]}$. Comparing with the cellular communication, D2D communication only occupies half of the spectrum resources. In addition, the users whose distance is closer can use D2D communication to reduce the transmission power ${ }^{[24,26]}$ and save energy. Therefore, D2D communication will probably become the key technology ${ }^{[23 \sim 27]}$ of mobile communications in the future. Of course, the interference between the cellular network communication system and D2D communication is a key problem, as well as the interference between the D2D users. In order to realize the whole benefit of direct communication, a series of power control and interference suppression methods can be used to realize the maximization of the interests of communication to meet the demands of users.

D2D communication is applicable to the local business, so if the base station reserves resources for a $\mathrm{D} 2 \mathrm{D}$ communication at the front end, the effective utilization of resources is low, especially when the probability of D2D is low. When common users and D2D users share the same resources, it will produce some interference in the community, resulting in the loss of performance. When this problem occurs, D2D users must control their transmit power properly from the perspective of ensuring the performance of the original cellular users. However, if the D2D mobile communication user is close to the cellular user who uses the same resources, the interference suppression effect of D2D user will be not ideal. It is noteworthy that the reason for this result is due to the huge difference of transmission power between the base station and the user.

One of the main problems of D2D communication is the interference caused by the resources reusing of cell users. When the D2D communication reuses uplink resources in the system, the 
base station will be interfered by the $\mathrm{D} 2 \mathrm{D}$ communication. When the $\mathrm{D} 2 \mathrm{D}$ communication reuses downlink resources in the system, the users of downlink will be interfered by the D2D communication. And the location of interfered downlink users depends on the short-term scheduling of the base station. Therefore, the users who interfered by D2D transmission ${ }^{[28]}$ may be any users of the community.

\section{POWER CONTROL SCHEME OF D2D COMMUNICATION}

Due to the reuse of the spectrum, D2D communication must have proper control, because serious interference may violate the original intent of D2D communication. PC (Power control) can suppress co-channel interference and optimize the performance of the system, which is an effective way to achieve the personal QoS requirements. In recent years, domestic and foreign research institutions have carried out the research of power control and made some progress. Figure 5 is the comparison chart before and after the implementation of power control scheme.

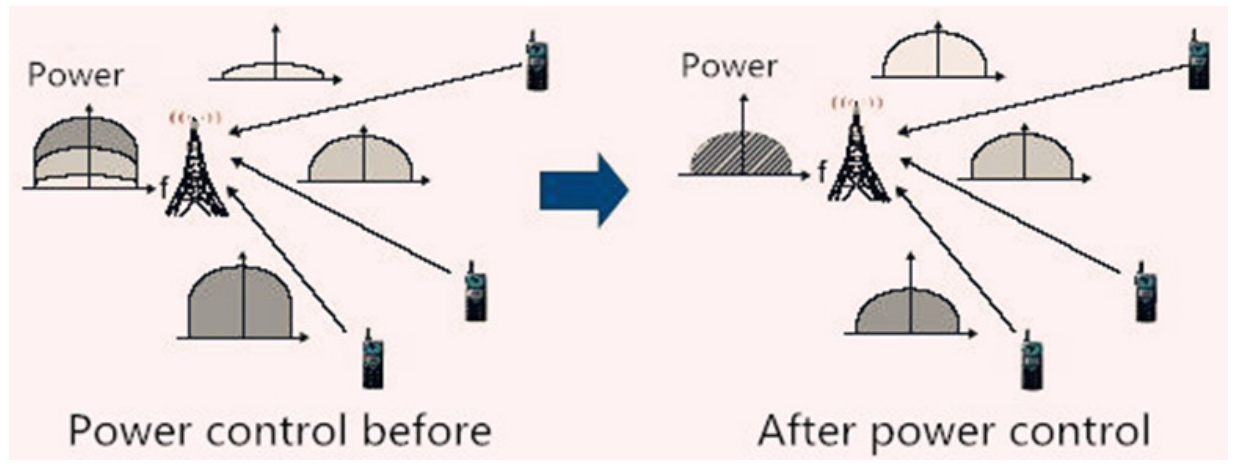

Figure 5. Comparison of power control before and after power control

Since the quality and requirements of cellular network ${ }^{[27-28]}$ terminal through communication are different, the scheme of power control is flexible and various, but the main purpose is to suppress the interference. In this paper, we mainly introduce three schemes of power control.

\subsection{Distributed Power Control}

There are two main types of DPC (Distributed power control) scheme: TPC (Target-tracking DPC) and OPC (Opportunistic DPC) ${ }^{[29]}$. In the TPC scheme, once the actual level of SNR (Signal to noise ratio) level is different with our setting SNR target, the transmission power will be adjusted to target power value of $\mathrm{SNR}^{[30]}$. In short, while achieving the target SNR level, it can also achieve a minimum total power consumption ${ }^{[31]}$.

In the OPC scheme, a low SINR (Signal to interference and noise ratio) level can't meet the requirements of QoS, and a high SINR level will lead to system capacity shrink ${ }^{[32]}$. OPC puts forward a SIP (Signal interference product) ${ }^{[33]}$ which uses the diversity of multi-user conflict link to detect the transmission desire of users. If the transmission power is large, a user can reduce his target SINR to prevent disproportionate power consumption, otherwise, the user can increase his target SINR to improve the utilization of radio resources by using limited channel conditions. OPC can avoid power emission, and every user has the opportunity to set their own reasonable SINR target to enhance the capacity of the system. 


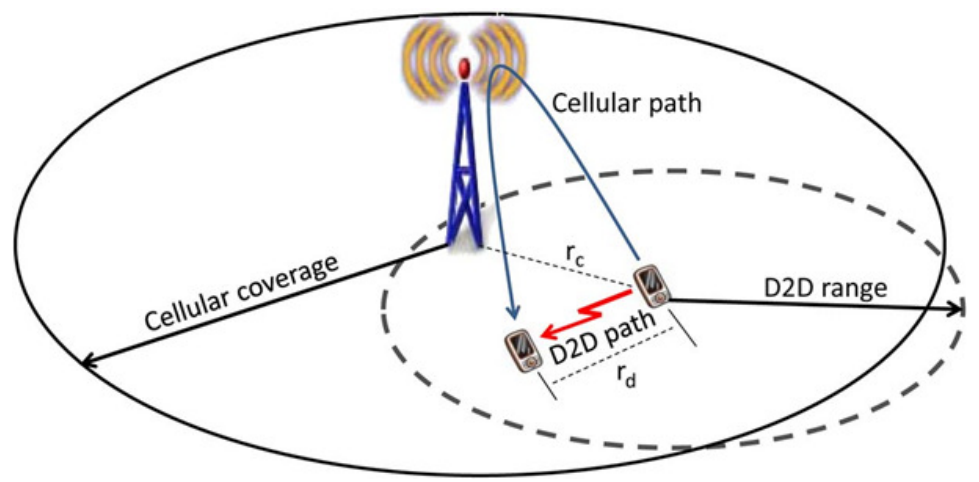

Figure 6. D2D communication under cellular network

As shown in Figure 6, we can take full use of interference avoidance algorithm to establish the constraint model of power control ${ }^{[34]}$ and set the parameters of cellular network coverage range, antenna gain, noise coefficient and power spectral density, etc. Also, we can set communication distance between two terminals, SNR, transmission power threshold, and adopt the bias scheme and admission control to analogy the two terminal communications of cellular network. We can verify the convergence and performance of DPC scheme ${ }^{[35]}$ through the system simulation. And finally, through avoiding invalid power consumption and maintaining the feasibility of the system, we can achieve the purpose of power control and interference suppression.

\subsection{Joint Control of Power and Rate}

The joint control ${ }^{[36,38]}$ of transmission power and rate ${ }^{[36-37]}$ can maximize the speed of cellular communication, and ensure the quality of D2D communication services at the same time. When a pair of D2D users share cellular network resources, SIC (Successive interference cancellation) and SUD (Single user detection) ${ }^{[39]}$ can be realized. The SIC is beneficial when cellular users have strong interference to the D2D receiver. When the interference is weak, D2D receiver can process the noise directly. So for a weak interference, the transmission rate and the transmission power of the cellular communication don't need to be controlled. D2D communication under cellular network is showed in Figure 5, when the cellular network users have strong interference to the $\mathrm{D} 2 \mathrm{D}$ receiver, the transmission power optimization strategy will be implemented to control the transmission rate. And when the interference is weak, the transmission rate optimization strategy will be implemented to control the transmission power. According to the different scenarios and users requirements, a similar optimal algorithm also can be set up on the basis of this scheme to find out the optimal value of the power and rate to meet respective demands.

\subsection{Joint Control of Power and Beam Forming}

Considering the characteristics of D2D and cellular network, the scheme based on SVM (Support vector machine $)^{[40]}$ can be used to solve the problem of constrained optimization existing in both cellular network user terminal and the D2D user terminal ${ }^{[4 \sim 43]}$. SVM is a supervised learning algorithm that uses known or similar scenes and functions to identify unknown or similar situations. Firstly, it transforms the constrained optimization problem into the minimization of the total power of all the terminals, which satisfies both the service quality requirement and the interference level. And then it uses the existing statistical channel state information ${ }^{[44-45]}$ to put 
forward an approximate method for releasing the constraint conditions. We use SVM algorithm to solve the optimization problem of transmission power and beam forming weight vector for each user $^{[46-48]}$. Then we can get the EC (Ergodic capacity) and ASER (Average symbol error rate) of the ergodic capacity of each user in the network. According to different scenario requirements, the purpose of power control can be achieved through the reasonable performance analysis of the simulation.

\section{GRAPH THEORY AIDED D2D}

Aiming at the interference in the uplinks of a system of multiple cells with co-existing of D2D users and cellular users, Yang etc. proposed a scheme of resources allocation among multiple cells based on interference graph and the best binary map matching ${ }^{[49]}$. In their scheme, people can obtain a weighted un-directional interference graph of D2D users and cellular users according to characteristics of uplinks, and distribute users with biggest interference into different clusters according to the interference graph. Different cluster has different sub-carrier and people can allocate resource blocks by Hungarian algorithm. The performance of this scheme is close to that of the exhaustive search scheme, but its complexity is much lower. And comparing to the scheme of fractional frequency reuse ${ }^{[50]}$, the proposed scheme can improve the entire system capacity by $19.78 \%{ }^{[49]}$.

Zhang etc. established an interference graph based on links of D2D and cellular users, and provided a resource allocation algorithm according to this interference graph. For their scheme, the capacity performance is close to the exhaust algorithm, and their scheme has lesser complexity ${ }^{[1]}$. In their scheme, there is a hypothesis that the base station knows the gain of each communication links and interference links in its coverage. This information is important for spectrum allocation between cellular users and D2D users.

In order to transmit multi-media video signal by D2D links, Zhang etc. used QoS as a key to evaluate whether this transmission is success or not. All users calculate the value of mean opinion score (MOS) according to quality of experience and send the results of calculation to video provider. Then this provider builds a weighted directed graph according to network topology and MOS of each possible connection ${ }^{[52]}$. Different from scheme of building conflict graph and weighted graph, Li etc. proposed an optimal method based on dynamic graph when considering the multi-hop D2D links and mobile nodes in massive grid $^{[53]}$. Aiming at the problem of transfer order among D2D communications in a time division multiplexing networks, researchers utilized min-cut graph partitioning and colouring principle to allocate resources. They planned the order of transmission between D2D links and also cellular links to minimizing total jamming in the networks ${ }^{[54]}$. Li etc. built a resource reuse graph and a conflict graph and change network connectivity optimization problem to the second smallest maximizing problem. They also adopted colouring theory for resources multiplexing and got the system capacity of D2D and cellular communications ${ }^{[55]}$.

Al-Hourani etc. used stochastic geometry study on D2D communication as a disaster relief solution. Base stations are easily to be damaged in kind of disasters. If mobile equipment (EU) can communicate with each other directly without the help of base stations, it will be convenient for disaster relief and post-disaster reconstruction ${ }^{[56]}$. In these situations, certain EUs can be used as low-power relay nodes, and build multiple links by D2D. 
By setting a guard distance, Ni etc. established a mode for pairs of co-existing D2D communications in the same cell. They considered the requirements of cellular main users and also D2D users. When uplink resources are reused, they set the guard distance between cellular main users and D2D users by geometric method ${ }^{[57,58]}$. In the help of guard distance, people can find the upper bound of system throughput, and this idea provides reference for the layout of D2D underlaying the cellular networks.

\section{CONCLUSIONS}

D2D communication may alleviate cellular network traffic, reduce the load on the local network, and increase the transmission rate. When UEs intercommunication, they can reduce the power consumption of the battery of the mobile terminal, thereby improving the robustness of the network infrastructure breakdown. Emergency communications can be used for earthquake rescue instead of damaged infrastructure. We consider the use of game theory approach, where D2D users are seen as the players. The players are self-interest, and they complete to maximize their own battery lifetime. We constructed resource allocation game and analysed the best response, Nash equilibrium and Pareto efficiency of the game. The players may create externality when they selfishly occupation radio resources, leading to declination in the quality of cellular communications. So we modify rules of the game by adding pricing as penalty and made a resource auction.

It is expected that communication system will become high-speed, large capacity, but the spectrum resource which can be used in mobile communication is very limited. So taking a full and efficient use of spectrum resources in the communication system is becoming more and more important in the future. D2D communication is applicable in licensed bands, comparing with WLAN, Bluetooth and other applications in communication technology of unlicensed bands; it has the advantages like interference control. Although the D2D communication under the resource reuse mode further improves the spectrum efficiency of the cellular network, it brings us new problems. Currently, researches of power control and interference suppression are performing continuously by domestic and foreign academic fields, various power control schemes are emerging in endlessly. Though the interference environment of cellular network terminal through communication is complex, through a series of experiments, it is found that the power control is an effective way to restrain the interference. It can improve the communication rate of the whole cellular network, and not only ensure the quality of the user service but also decrease the energy consumption to provide users with a more stable and controllable communication environment.

Kinds of dense and underlaying networks will co-exist with the cellular one in the future, in which the D2D communication and femtocell in the five generation LTE (Long term evolution) nets will become more and more popular in the requirement of high speed data transfer.

\section{ACKNOWLEDGEMENTS}

The work is supported by Natural Science Foundation of China (61661018), Natural Science Foundation of Hainan Province (20166210), Key Project on Science Research of Higher School from Hainan Department of Education (Hnky2016ZD-5), High Tech. of Key Research and Development Project of Hainan Province (ZDYF2016010), Scientific Research Foundation of Hainan University (kyqd1536).Hui Li is the corresponding author. 
International Journal of Computer Networks \& Communications (IJCNC) Vol.8, No.5, September 2016

\section{REFERENCES}

[1] K. Doppler, M. Rinne, C. Wijting, C. Ribeiro, K. Hugl, "Device-to-device communication as an underlay to LTE-advanced networks", IEEE Communiction Magazine, 47(12), 42-49 (2009)

[2] P. Janis, V. Koivunen, C. B. Ribeiro, K. Doppler, "Interference-avoiding MIMO schemes for deviceto-device radio underlaying cellular networks", IEEE $20^{\text {th }}$ International Symposium on Personal, Indoor and Mobile Radio Communications, pp. 2385-2389, Tokyo, Sept. (2009)

[3] T. Peng, Q. Lu, H. Wang, S. Xu, W. Wang, "Interference avoidance mechanisms in the hybrid cellular and device-to-device systems", IEEE $20^{\text {th }}$ International Symposium on Personal, Indoor and Mobile Radio Communications, pp. 617-621, Tokyo, Sept. (2009)

[4] P. Janis, V. Koivunen, C. Ribeiro, J. Korhonen, K. Doppler, K. Hugl, "Interference-aware resource allocation for device-to-device radio underlaying cellular networks", Proceedings of IEEE Vehicular Technology Conference, 2009-Spring, Barcelona, April (2009)

[5] M. Zulhasnine, C. Huang, A. Srinivasan, "Efficient resource allocation for device-to-device communication underlaying LTE network", IEEE $6^{\text {th }}$ International Conference on Wireless and Mobile Computing, Networking and Communications, pp. 368-375, Niagara Falls, Oct. (2010)

[6] S. Xu, H. Wang, T. Chen, Q. Huang, T. Peng, "Effective interference cancellation scheme for deviceto-device communication underlaying cellular networks", Proceedings of IEEE Vehicular Technology Conference, 2010-Fall, Ottawa, Sept. (2010)

[7] C. H. Yu, K. Doppler, C. Ribeiro, O. Tirkkonen, "Resource sharing optimization for D2D communication underlaying cellular networks", IEEE Transactions on Wireless Communication 10(8), 2752-2763 (2011)

[8] J. Bae, E. Beigman, R. Berry, M. L. Honig, R. Vohra, "Sequential bandwidth and power auctions for distributed spectrum sharing”, IEEE Journal on Selected Areas in Communications, 26(9), 1193-1203, (2008)

[9] A. Das, D. Grosu, "Combinatorial auction-based protocols for resource allocation in grids", Proceedings of IEEE International Parallel and Distributed Processing Symposium, April (2005)

[10] H. C. Lau, S. F. Cheng, T. Y. Leong, J. H. Park, Z. Zhao, "Multi-period combinatorial auction mechanism for distributed resource allocation and scheduling", IEEE/WIC/ACM International Conference on Intelligent Agent Technology, pp. 407-411, Fremont, Nov. (2007)

[11] P. Cramton, Y. Shoham, R. Steinberg, "Combinatorial Auctions", MIT Press, Cambridge (2005)

[12] A. Pikovsky, "Pricing and bidding strategies in iterative combinatorial auctions", Ph. D. Dissertation, Munich, Germany (2008)

[13] M. Bichler, P. Shabalin, A. Pikovsky, "A computational analysis of linear price iterative combinatorial auction formats", Information Systems Research, 20(1), 33-59, (2009)

[14] B. Wang, Z. Han, K. J. R. Liu, "Distributed relay selection and power control for multiuser cooperative communication networks using buyer/seller game", $26^{\text {th }}$ IEEE International Conference on Computer Communications, pp. 544-552, Barcelona, May (2007)

[15] J. Zhang, Q. Zhang, "Stackelberg game for utility-based cooperative cognitive radio networks", Proceedings of ACM International Symposium on Mobile ad hoc Networking and Computing, pp. 2332, New Orleans, May (2009)

[16] M. Bloem, T. Alpcan, T. Basar, "A Stackelberg game for power control and channel allocation in cognitive radio networks", Proceedings of International Conference on Performance Evaluation Methodologies and Tools, Dresden, Oct. (2007)

[17] R. Rao, S. Vrudhula, D. N Rakhmatov, "Battery modelling for energy aware system design", Computer, 36(12), 77-87 (2003)

[18] A. Asadi, Q. Wang, V. Mancuso, “A survey on device-to-device communication in cellular networks", IEEE Communication Surveys and Tutorials, 16(4), 1801-1819, Apr. (2014)

[19] G. Fodor, E. Dahlman, G. Mildh, Parkvall, N. Reider, G. Miklos, Z. Turanyi, "Design aspects of network assisted device-to-device communications", IEEE Communication Magazine, 50(3), 170177, March (2012)

[20] L. Lei, Z. Zhong, C. Lin, X. Shen, "Operator controlled device-to-device communications in LTEAdvanced networks”, IEEE Wireless Communications, 19(3), 96-104, June (2012) 
[21] 3GPP SP-110638, "Study on proximity-based services. 3GPP TSG SA Plenary document", Fukuoka, Japan, 19-21 Sept. (2011)

[22] M. Corson, R. Laroia, J. Y. Li, V. Park, T. Richardson, G. Tsirtsis, "Toward proximity aware internetworking", IEEE Wireless Communications, 17(6), 26-33, (2010)

[23] P. Janis, C. H. Yu, K. Doppler, C. Ribeiro, C. Wijting, K. Hugl, O. Tirkkonen, V. Koivunen, "Deviceto-device communication underlaying cellular communications systems", International Journal of Communications, Network \& System Sciences, 2(3),169-178, (2009)

[24] C. Wijting, C. Ribeiro, K. Doppler, M. Rinne, K, Hugl, "Device-to-device communication as an underlay to LTE-advanced networks", IEEE Communications Magazine, 47(12),42-49, (2009)

[25] B. Kaufman, B. Aazhang, "Cellular networks with an overlaid device to device network", IEEE $42^{\text {nd }}$ Asilomar Conference on Signals, Systems and Computers, 1537-1541,(2008)

[26] G Fodor, E. Dahlman, G. Mildh, S. Parkvall, N. Reider, "Design aspects of network assisted deviceto-device communications", IEEE Communication Magazine, 50(3), 170-177, March (2012)

[27] K. Doppler, M. P. Rinne, P. Janis, C. Ribeiro, "Device-to-device communications: Functional prospects for LTE-Advanced networks", IEEE International Conference on Communications Workshops, 1-6, (2009)

[28] S. J. Liu, B. Wang, W. Wang, "A novel interference coordination algorithm for LTE system", Computer Engineering and Applications, 50(3), 207-210, (2014)

[29] R. Tang, J. Zhao, Q. U. Hua, "Distributed power control for energy conservation in hybrid cellular network with device-to-device communication", Wireless Communication Over Zigbee for Automotive Inclination Measurement China Communications, 11(3), 27-39, (2014)

[30] G. Foschini, Z. Miljanic, "A simple distributed autonomous power control algorithm and its convergence', IEEE Transactions on Vehicular Technology, 42(4), 641-646, (1993)

[31] S. Grandhi, J. Zander, "Constrained power control in cellular radio systems', Proceedings of $44^{\text {th }}$ Vehicular Technology Conference, Stockholm, Sweden, 824-828, (1994)

[32] C. Sung, K. Leong, "A generalized framework for distributed power control in wireless networks", IEEE Transactions on Information Theory, 51(7), 2625-2635, (2005)

[33] K. Leong, C. Sung, “An opportunistic power control algorithm for cellular network", IEEE/ACM Transactions on Networking, 14(3),470-478, (2006)

[34] N. Lee, X. Lin, J. G. Andrews, R. W. Heath Jr, "Power control for D2D underlaid cellular networks: modeling, algorithms and analysis", IEEE Journal on Selected Areas in Communication, 33(1), 1-13, Jan. (2015)

[35] R. Yates, “A framework for uplink power control in cellular radio systems”, IEEE Journal on Selected Areas in Communications, 13(7),1341-1347, (1995)

[36] H. Elsawy, E. Hossain, M. S. Alouini, "Analytical modelling of mode selection and power control for underlay D2D communication in cellular networks", IEEE Transactions on Communication, 62(11), 4147-4161, Nov. (2014)

[37] J. Wang, D. Zhu, C. Zhao, J. C. F. Li, M. Lei, "Resource sharing of underlaying device to device and uplink cellualr communications", IEEE Communication Letters, 17(6), 1148-1151, June (2013)

[38] H. Song, J. Ryu, W. Choi W, R. Schober, "Joint Power and Rate Control for Device-to-Device Communications in Cellular Systems", IEEE Transactions on Wireless Communications, 14(10), 1-1, (2015)

[39] N. K. Pratas, P. Popovski, "Underlay of low-rate machine-type D2D links on downlink cellular links", IEEE International Conference on Communications Workshops, 1-5, June (2014)

[40] V. N. Vapnik, "Statistical learning theory", New York: Wiley, (1998)

[41] M Lin, J. Ouyang, W. P. Zhu, "Joint beamforming and power control for device-to-device communications underlaying cellular networks", IEEE Journal on Selected Areas in Communications, 34(1),138-150, (2016)

[42] S. Theodoridis, K. Slavakis, I. Yamada, "Adaptive learning in a world of projections", IEEE Signal Processing Magazine, 28(1), 97-123, Jan. (2011)

[43] C. C. Gaudes, I. Santamatia, J. Via, E. M. Gomez, T. S. Paules, "Robust array beamforming with sidelobe control using support vector machines", IEEE Transaction on Signal Processing, 55(2), 574$584,(2007)$ 
[44] M. Li, M. Lin, Q. Yu, W. P. Zhu. L Dong, "Optimal beamformer design for two hop MIMO AF relay networks over Rayleigh fading channels," IEEE Journal on Selected Areas in Communications, 30(8), 1402-1414, Sept. (2012)

[45] O. N. Alrabadi, E. Tsakalaki, H. Huang, G. F. Pedersen, "Beamforming via large and dense antenna arrays above a clutter," IEEE Journal on Selected Areas in Communications, 31(2), 314-325, Feb. (2013)

[46] O. N. Alrabadi, E. Tsakalaki, H. Huang, G. F. Pedersen, "Beamforming via large and dense antenna arrays above a clutter," IEEE Journal on Selected Areas in Communications, 31(2), 314-325, Feb. (2013)

[47] A. Amiri, "Bandwidth reservation and adaptation in wireless communication networks," International Journal of Computer Networks and Communications, 8(1), 21-33, Jan. (2016)

[48] S. Kuribayashi, "Resource allocation method for cloud computing environments with different service quality to users at multiple access points," International Journal of Computer Networks and Communications, 7(6), 33-51, Nov. (2015)

[49] Y. Yang, X. W. Liao, Z. Z. Gao, G. M. Lv, "A resource allocation scheme using theory for D2D communication in multi-cell heterogeneous cellular networks," Journal of Xi' an Jiaotong University, 48(10), 22-28, Oct. (2014)

[50] H. S. Chae, J. Gu, B. G Choi, M. Y. Chung, "A resource allocation scheme using theory for D2D communication in multi-cell heterogeneous cellular networks," 17th Asia-Pacific Conference on Communications, pp. 22-28, Sabah, Malaysia, Oct. (2011)

[51] R. Q. Zhang, X. Cheng, L. Q. Yang, B. L. Jiao, "Interference-aware graph based resource sharing for device-to-device communications underlaying cellular networks," IEEE Wireless Communications and Networking Conference, pp. 140-145, (2013)

[52] A. Q. Zhang, J. X. Chen, L. Zhou, S. Yu, "Graph theory based QoE-driven cooperation stimulation for content dissemination in device-to-device communication," IEEE Transactions on Emerging Topics in Computing, 99, (2015)

[53] Y. Li, C. M. Song, D. P. Jin, S. Chen, "A dynamic optimization framework for multihop device-todevice communication underlaying cellular networks," IEEE Wireless Communications, 21(5), 52-61, (2014)

[54] Z. Uykan, R. Jantti, "Transmission-order optimization for bidirectional device-to-device(D2D) communications underlaying cellular TDD networks- A graph theoretic approach," IEEE Journal on Selected areas in communications, 34(1), 1-14, (2016)

[55] J. Li, J. B. Song, Z. Han, "Network connectivity optimization for device-to-device wireless system with femtocell," IEEE Transactions on Vehicular Technology, 62(7), 3098-3108, (2013)

[56] A. Al-Hourani, S. Kandeepan, A. Jamalipour, "Stochastic geometry study on device to device communication as a disaster relief solution," IEEE Transaction on Vehicular Technology, 2(4), 358382, (2015)

[57] M. M. Ni, L. Zhang, F. Tong, J. P. Pan, L. Cai, "A geometrical-based throughput bound analysis for device-to-device communications in cellular networks," IEEE Journal on Selected Areas in communications, 33(1), 100-110, (2015)

[58] M. M. Ni, J. P. Pan, L. Cai, "Geometrical-based throughput analysis of device-to-device communications in a sector-partitioned cell," IEEE Transactions on Wireless Communications, 14(4), 2232-2244, (2015)

\section{AUTHORS}

Fa-Bin Li received B.E. degree from Chinese people's Liberation Army Equipment Institute in 2002. He is now a senior engineer and chief engineer in communication terminal of 63812 Troop of PLA. His main research areas are wireless communication, satellite networks and mobile computing.

Peng-Xiang Li received B.E. degree from Jingchu University of Technology in 2015. He is now a graduate student in Hainan University. His research areas are interference control and management in D2D communications and in maritime communications. He is a student member of IEEE.

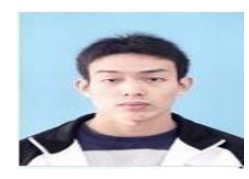


International Journal of Computer Networks \& Communications (IJCNC) Vol.8, No.5, September 2016

Ya-Fei Yang received B.E. degree from Changchun University of Science and Technology, China in 2015. He is now a graduate student in Hainan University. His research areas are power control in D2D communications and in maritime communications.

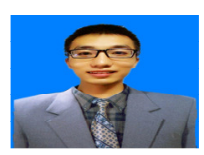

Hui Li received B.E., M. E., and D. E. degrees from Harbin Institute of Technology (HIT), China, in 2000, 2002 and 2006 respectively. He worked as a post-doctoral and an associate professor in Shenzhen graduate school of HIT from Dec. 2005 to Feb. 2009. He joined Zhejiang University from March 2009 to Nov. 2014. He has been a visiting scholar in National Institute of Standard and Technology of DoC of USA from Nov.

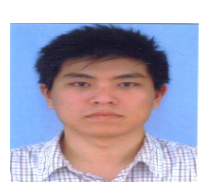
2012 to Nov. 2013. He is currently working as a full professor in School of Information Science and Technology of Hainan University. His researches include advanced wireless networks, maritime communication, space communication. He is a senior member of IEEE and a member of IEICE. 